\title{
Systematical nucleon-induced optical model analysis for medium and heavy nuclei with coupled-channel framework
}

\author{
S. Kunieda ${ }^{1, a}$, S. Chiba ${ }^{1}$, K. Shibata ${ }^{1}$, A. Ichihara ${ }^{1}$, and E.Sh. Sukhovitskiî ${ }^{2}$ \\ 1 Japan Atomic Energy Agency, Tokai-mura, Naka-gun, Ibaragi-ken 319-1195, Japan \\ 2 Joint Institute for Energy and Nuclear Research, 220109 Minsk-Sosny, Belarus
}

\begin{abstract}
Optical model analyses have been carried out for nucleon-induced reactions on medium and heavy nuclei from $1 \mathrm{keV}$ up to $200 \mathrm{MeV}$. The coupled-channels method based on the rigid-rotator model has been employed as an initial approach. It has been found that the coupled-channels analyses are indispensable to obtain a global formulation of optical potential. In addition, the coupled-channels calculation based on the soft-rotator model has been applied to analyses for various even-even fission product isotopes. They reproduced measured cross sections better than the rigid-rotator model in many cases. Though the latter is our ongoing study, we show the advantage of coupled-channels calculations and the applicability of the soft-rotator model.
\end{abstract}

\section{Introduction}

There is no doubt that the optical model plays an important role in evaluating/predicting scattering and absorption cross sections above the resolved resonance region. Since it also provides transmission coefficients required in the statistical model, the optical model potential must be determined precisely in the nuclear data evaluation. The optical potential varies with projectiles, target nuclei and collision energies and it is generally optimized on experimental data of total, absorption and scattering cross sections. However, measured data are very scarce in general, except for some special nuclei, namely, they are sparse over wide nuclear-mass and energy ranges. Therefore, a problem arises, e.g., in doing nuclear data evaluations for the bulk of fission products. Therefore, it might be required to derive an optical potential which predicts cross sections with high accuracy for various nuclei over a wide energy range. Until now, a lot of studies have been devoted in order to obtain such potential. The most useful one was done by Koning and Delaroche [1]. However, the spherical shape was assumed in their analyses, where a consideration of deformed shapes and the coupled-channels calculation should give more actual answers. In fact, it is widely known that there exist many deformed nuclei in their ground and/or excited states.

In this study, nucleon-induced optical model analyses are carried out in the coupled-channels frameworks for various nuclei $(26 \leq Z \leq 92)$ in the energy range from $1 \mathrm{keV}$ up to $200 \mathrm{MeV}$. The coupled-channels method based on the rigidrotator model (RRM-CC) is employed as an initial approach, in order to survey global tendencies of optical potential parameters. Then, we apply the soft-rotator model (SRM) [2] to the coupled-channels calculations for various even-even fission product nuclei. We show the advantage of the coupledchannels calculation and applicability of the SRM in this work. We also would like to discuss about systematics for groundstate deformation within the framework of the SRM-CC.

${ }^{a}$ Presenting author, e-mail: kunieda.satoshi@jaea.go.jp

\section{Brief descriptions for systematic optical model potential}

Though collective motions of a target nucleus are ascribed to rotation and/or vibration in general, it is not easy to explicitly know them except for some typical cases. Accordingly, the coupled-channels method based on the rigid-rotator model is uniformly adopted as an initial approach in order to simplify the problem. The functional forms of Sukhovitskiĩ-Chiba [5] are used to express the energy dependences of optical potential depths. They are written as follows for real volume, imaginary surface and imaginary volume terms, respectively (note that the spin-orbit terms are taken from those of Koning and Delaroche [1]):

$$
\begin{aligned}
V_{R}\left(E^{\dagger}\right) & =\left(V_{R}^{0}+V_{R}^{1} E^{\dagger}+V_{R}^{2} E^{\dagger 2}+V_{R}^{3} E^{\dagger 3}+V_{R}^{D I S P} e^{-\lambda_{R} E^{\dagger}}\right) \\
& \times\left[1+\frac{1}{V_{R}^{0}+V_{R}^{D I S P}}(-1)^{Z^{\prime}+1} C_{\text {viso }} \frac{N-Z}{A}\right] \\
& +C_{\text {coul }} \frac{Z Z^{\prime}}{A^{1 / 3}} \varphi_{\text {coul }}\left(E^{\dagger}\right) \\
W_{D}\left(E^{\dagger}\right) & =\left[W_{D}^{D I S P}+(-1)^{Z^{\prime}+1} C_{\text {wiso }} \frac{N-Z}{A}\right] \\
& \times e^{-\lambda_{D} E^{\dagger}} \frac{E^{\dagger 2}}{E^{\dagger 2}+W I D_{D}^{2}} \\
W_{V}\left(E^{\dagger}\right) & =W_{V}^{D I S P} \frac{E^{\dagger 2}}{E^{\dagger 2}+W I D_{V}^{2}}
\end{aligned}
$$

It should be noted that these forms pre-consider isospin dependence and Coulomb correction. They allow us to carry out optical model analyses both for incident neutrons and protons simultaneously. Also, the symmetric terms describe major parts of the isotopic variation of potential depths. The symbols $E^{\dagger}$ and $Z^{\prime}$ denote projectile energy relative to the Fermi energy and the charge number of projectile, respectively. The Coulomb term $\varphi_{\text {coul }}$ is treated as a minus derivative of the other terms of real volume strength $V_{R}$ with respect to $E$. 
The values of $V_{R}^{(0-3)}, V_{R}^{D I S P}, W_{D}^{D I S P}, W_{V}^{D I S P}, \lambda_{R}, \lambda_{D}, C_{\text {viso }}$, $C_{\text {wiso }}$ and $C_{\text {coul }}$ are adjustable parameters to be optimized phenomenologically.

These parameters are searched for along with deformation parameters so as to reproduce experimental data of total, total-reaction, elastic and inelastic scattering cross sections concentrating on some specific nuclei. We chose 54,56,58,nat $\mathrm{Fe},{ }^{58,60,62,64, \text { nat }} \mathrm{Ni},{ }^{63,65, \text { nat }} \mathrm{Cu},{ }^{89} \mathrm{Y},{ }^{90,92,94, \text { nat }} \mathrm{Zr},{ }^{93} \mathrm{Nb}$,

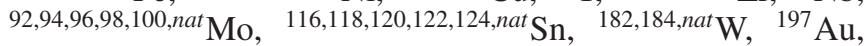
${ }^{208, n a t} \mathrm{~Pb},{ }^{209} \mathrm{Bi},{ }^{232} \mathrm{Th}$ and ${ }^{238} \mathrm{U}$ for local analyses. The $\mathrm{CC}$ computations and parameter searches are done with the OPTMAN code [3] which considers the relativistic kinematics. The program POD [7] is supplementarily adopted in order to calculate compound elastic scattering cross sections. We obtain a systematic nucleon optical potential which is formulated by simple forms over ranges $1 \mathrm{keV} \leq \mathrm{E} \leq 200 \mathrm{MeV}$ and $26 \leq Z \leq 92$. Its validities are confirmed in our global surveys, e.g., s- and p-wave neutron strength functions [8]. It is found that the coupled-channels calculations are indispensable to achieve a global description of cross sections over wide energy and nuclear mass ranges [4]. Though it gives better results than those of the spherical model, there were deficiencies in precise descriptions of measured data for some cases, i.e., neutron cross sections below incident energy of $\sim 1 \mathrm{MeV}$ where the collective properties tended to largely influence cross sections. We try to survey whether the SRM-CC solves the problem or not.

\section{SRM-CC analyses for even-even isotopes}

It is very important to consider the collective natures of a target nucleus in the optical model analysis. In the RRM-CC analyses, these natures are expressed just by the rotation of the axial deformed shape. The manner, however, might be an extreme approach except for typical deformed nuclei, because it confuses with vibrational property. Actually, it is not easy to precisely describe the characteristics of nuclei with a simple framework because they are quite variant. According to those facts, we employ the SRM-CC which is expected to treat collective properties of nuclei more precisely. Before now, the model has been applied to nucleon-nucleus interaction studies for some of the major nuclei, and its applicability has been confirmed step by step (see e.g., refs $[2,5,6,9]$ ). Now, we are trying to apply the model to various nuclei over the fission product mass range. In this study, We carry out the analyses following four procedures.

\subsection{Analyses of low-lying collective level structures}

The SRM is the model which can predict major collective properties such as elasticities and non-axial deformations of a nucleus from the experimentally known excited level structure. It can also provide coupling strengths among the ground and excited states. The descriptions of the model are given in detail elsewhere $[2,3,5,6]$. The SRM analyses are carried out for various even-even fission product nuclei. The low-lying level data are taken from the latest versions of the Nuclear Data Sheets, and the ground state (G.S.) band,



Fig. 1. The collective level structure of ${ }^{100} \mathrm{Mo}$ which was obtained in the SRM analysis, together with the experimental one.

the $\beta_{2}$-vibrational $\left(\mathrm{n}_{\beta_{2}}=1\right), \gamma$-vibrational $(\mathrm{K} \approx 2)$ and the octupole negative parity $\left(\mathrm{n}_{\beta_{3}}=0\right)$ bands are used in the analyses. The level assignments are done by ourselves when those band structures were not well-known. The nuclear Hamiltonian parameters of collective motions (i.e., elasticities, non-axialities, mass parameters, equilibrium $\beta_{3} / \beta_{2}$ ratio) are determined so as to reproduce the low-lying level structures. The program SHEMMAN [3] is utilized for these analyses. Generally, both the quadrupole and the octupole modes are taken into account for the rotational-vibrational motion. In addition to them, the hexadecapole mode is also considered for typical deformed nuclei. We are forced to exclude some of the nuclei which had a closed magic shell from the analyses, because they never show typical collective level structure.

One of the results is shown in figure 1 for ${ }^{100} \mathrm{Mo}$. We have obtained the SRM Hamiltonian parameters which could reproduce the experimental band structure. Note that we can clearly see a $\gamma$-vibrational band. Also note that it is not a rare case for the medium weight nuclei.

\subsection{SRM-CC analyses for proton scattering}

The SRM-CC analyses have been carried out using our systematic optical potential and nuclear Hamiltonian parameters obtained in the SRM level structure analyses. The OPTMAN code enabled us to do the SRM-CC computations. The purpose of these analyses was to determine the values of ground state deformations. In doing the CC calculations, the values of quadrupole (hexadecapole) ground state deformation $\beta_{2}\left(\beta_{4}\right)$ have been searched for so as to reproduce measured proton-induced inelastic scattering differential cross sections of the first $2^{+}\left(4^{+}\right)$level excitation(s). Actually, they could not be derived from the SRM level structure analysis itself. In general, the ground state band $\left(0^{+}, 2^{+}, 4^{+} \ldots\right)$ 



Fig. 2. The calculated direct inelastic scattering differential cross sections for $\mathrm{p}+{ }^{100}$ Mo together with the experimental data [10-12].

and octupole $\left(3^{-}\right)$level are coupled in the present study. Though it was too weak excitation to be considered in many cases, the first $\gamma$-vibrational level $\left(2^{+}\right)$was also coupled when it yielded measurable inelastic scattering cross sections. In this case, the elasticity parameter of $\gamma$ vibration was determined so as to describe corresponding measured inelastic scattering cross sections, since we could not determine its elasticity parameter due to the difficulty of the level assignment in the SRM analysis. Of course, those calculations could be applied only to the cases where inelastic scattering experimental data were available: ${ }^{54,56} \mathrm{Fe},{ }^{58,60,62,64} \mathrm{Ni}, \quad{ }^{64,66,68,70} \mathrm{Zn},{ }^{70,72,74,76} \mathrm{Ge},{ }^{76,78,80,82} \mathrm{Se}$, ${ }_{96,98,100} \mathrm{Mo}, \quad{ }^{102} \mathrm{Ru}, \quad{ }^{104,106,108,110} \mathrm{Pd}, \quad 106,108,110,112,114,116 \mathrm{Cd}$, ${ }_{116,118,120,122,124} \mathrm{Sn},{ }^{144,150} \mathrm{Nd},{ }^{148,150,152,154} \mathrm{Sm},{ }^{160} \mathrm{Gd},{ }^{166} \mathrm{Dy}$, ${ }^{166,168} \mathrm{Er},{ }^{172,174,176} \mathrm{Yb},{ }^{178,180} \mathrm{Hf},{ }^{182,184} \mathrm{~W}$.

As an example, the calculated inelastic scattering cross sections are illustrated in figure 2 for ${ }^{100}$ Mo together with the experimental data [10-12]. It should be emphasized that the calculated cross sections of $3^{-}$excitation reproduce the data very well. The RRM-CC actually could not treat it by itself. The fact shows one of the advantages of the SRM-CC calculation, which was due to the coupling strength of the negative parity band. In table 1 , we also list the obtained equilibrium deformations $\beta_{2}$ and the effective deformations at $2_{1}^{+}$state $\left\langle 0_{\text {G.S. }}^{+}\left|\beta_{2}\right| 2_{1}^{+}\right\rangle$for ${ }^{96,98,100} \mathrm{Mo}$, and they are compared with the experimental values [17] which were deduced from the $\mathrm{B}(\mathrm{E} 2)$ analysis. Though the obtained equilibrium

Table 1. The obtained quadrupole deformations of equilibrium and $2_{1}^{+}$states for ${ }^{96,98,100} \mathrm{Mo}$, together with the experimental values [17].

\begin{tabular}{rccc}
\hline & $\beta_{2}$ & $\left\langle 0_{\mathrm{G} . S .}^{+}\left|\beta_{2}\right| 2_{1}^{+}\right\rangle$ & Experimental values \\
\hline${ }^{96} \mathrm{Mo}$ & 0.060 & 0.191 & $0.172( \pm 0.0016)$ \\
${ }^{98} \mathrm{Mo}$ & 0.027 & 0.174 & $0.168( \pm 0.0028)$ \\
${ }^{100} \mathrm{Mo}$ & 0.045 & 0.241 & $0.231( \pm 0.0022)$ \\
\hline
\end{tabular}

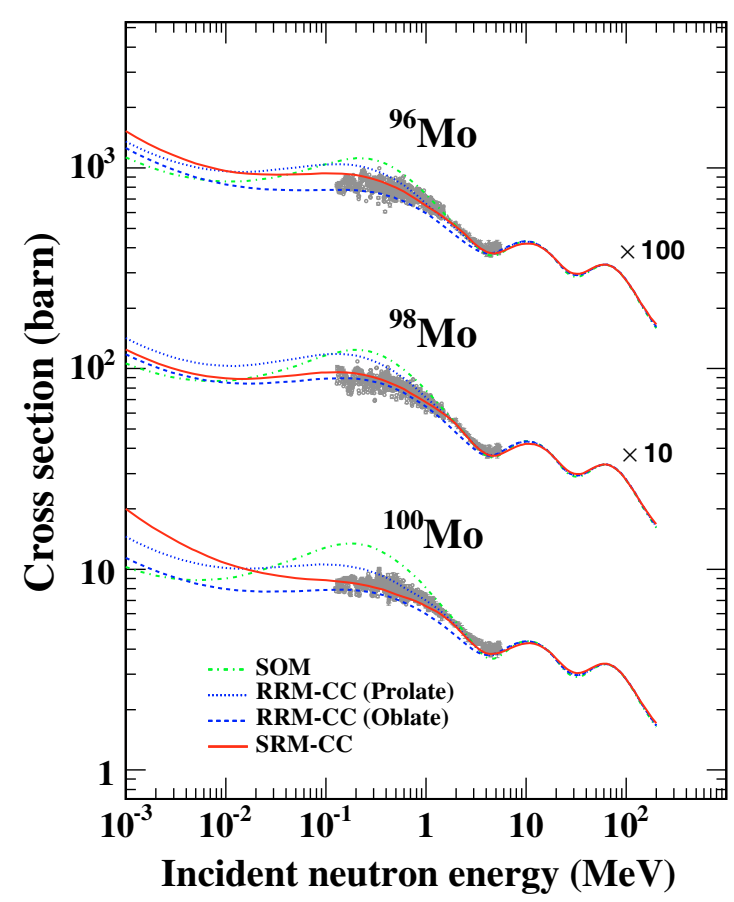

Fig. 3. The calculated neutron total cross sections for ${ }^{96,98,100} \mathrm{Mo}$ together with the experimental data [13-16].

deformations are quite small for those isotopes, our effective deformations are consistent with the measured ones.

\subsection{SRM-CC calculations for neutron cross sections}

The SRM-CC calculations were applied to neutron-induced reactions for various even-even fission product isotopes. Again, the optical potential was fixed to the systematics which was deduced in our global study based on the RRM-CC. Figure 3 presents the calculated neutron total cross sections for $96,98,100$ Mo together with measured data [13-16]. The SRM$\mathrm{CC}$ results are compared with the spherical model and the RRM-CC (both prolate and oblate) calculations. There are no clear differences between the model calculations above $10 \mathrm{MeV}$ while noticeable differences can be seen especially below $1 \mathrm{MeV}$. This means that the collective characteristics hardly affect cross sections in the high energy reaction, while the cross sections strongly depend on the collective characteristics in the low energy region. We can find that the spherical model apparently has a deficiency especially below $1 \mathrm{MeV}$ in evaluating cross sections systematically. On the other hand, the RRM-CC gives better results than spherical model. This finding suggests that the ground state is strongly coupled with the collective motions even in the Mo isotopes which are not supposed to be typical deformed nuclei. Here, it should be noted that the prolate/oblate assignments affect cross sections in the RRM-CC calculations. Also note that both calculations tend to overestimate/underestimate the experimental data. This fact might mean that the use of the RRM-CC is no longer a preferable approach in precise evaluations of those cases. The SRM-CC results agree with measured cross sections better than the RRM-CC calculations. It is substantially ascribed to the considerations of the equilibrium non-axialities 


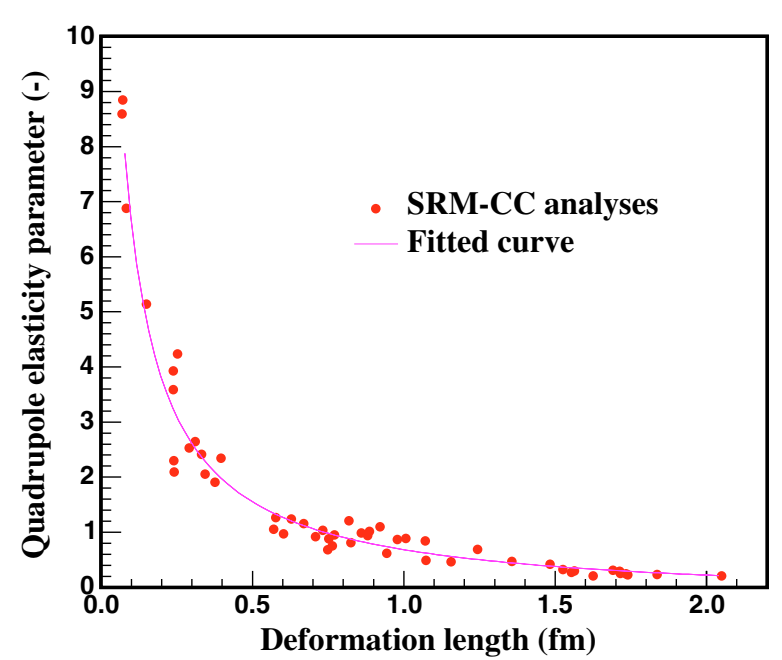

Fig. 4. The relationship between the ground state deformations and the elasticity parameters of quadrupole mode which is obtained by the SRM-CC analyses for various even-even nuclei $\left(R=1.21 A^{1 / 3}\right.$ was assumed for derivations of the deformation length).

which were predicted in the SRM level structure analyses. Actually we obtained large values of non-axiality parameters $\left(\gamma_{0} \sim \pi / 6\right)$ not only for Mo isotopes, but also for many fission product nuclei. Of course, it was also due to the elasticity parameters which consequently allowed us to obtain more realistic quadrupole deformations.

\subsection{Possibility of systematic treatment for the ground state deformations}

The quadrupole equilibrium deformation parameters obtained in the SRM-CC analyses should give more actual ground state deformations, because they are not confused with the amplitude of vibration, and also, they are already independent of the non-axial deformation. Actually, vibrational properties are completely described by the elasticity parameters of a nucleus. The asymmetric deformation was expressed by the non-axial parameters. We tried to investigate how the ground state deformations varied in correlations with the other parameters for various cases. As a result, it was found that there exists an obvious relationship between the quadrupole ground state deformations and the elasticity parameters as shown in figure 4 . Though it is a preliminary result, there is a possibility that we can know the ground state deformations to be used in $\mathrm{CC}$ calculation from the elasticity parameter which is derived from the SRM level structure analysis.

\section{Conclusions}

Nucleon-induced optical model analyses have been carried out for various medium and heavy nuclei over an energy range from $1 \mathrm{keV}$ to $200 \mathrm{MeV}$. The RRM-CC allowed us to describe global tendencies of optical potential by simple functional forms and the systematical parameters. Then we applied the SRM-CC to the analyses for various even-even fission product nuclei. The systematic optical potential plus the SRM-CC calculations showed better results than those of the RRM-CC. The good results of the SRM-CC are substantially due to the consideration of non-axial deformation which is obtained by the nuclear level structure analysis. Moreover, it was also found that there exists a clear correlation between the quadrupole elasticity parameter and ground state deformation. This means that the values of the ground state deformation are likely to be guessed by the results of SRM nuclear level structure analyses. We confirm that the SRM-CC is a useful approach to give more elaborate evaluations/predictions of nuclear data.

The authors would like to thank Drs Osamu Iwamoto and Nobuyuki Iwamoto of Japan Atomic Energy Agency for useful comments on this work.

\section{References}

1. A.J. Koning, J.-P. Delaroche, Nucl. Phys. A 713, 231 (2003).

2. E.Sh. Sukhovitskii et al., Phys. Rev. C 62, 044605 (2000).

3. E.Sh. Sukhovitskii et al., JAERI-Data/Code 2005-002 (2005).

4. S. Kunieda et al., J. Nucl. Sci. Technol. 44(6) (2007) (in press).

5. E.Sh. Sukhovitskii et al., J. Nucl. Sci. Technol. 39(8), 816 (2002).

6. S. Chiba et al., J. Nucl. Sci. Technol. 37(6), 498 (2000).

7. A. Ichihara et al., JAEA-Data/Code 2006-025 (2007).

8. K. Shibata et al. (these proceedings).

9. E.Sh. Sukhovitskii et al., Proc. of Int. Conf. on Nucl. Data for Sci. and Technol., Santa Fe, New Mexico, USA, 26 Sept.-1 Oct., 2004 (AIP, Melville, New York, 2005), pp. 1058-1061.

10. H.F. Lutz et al., Phys. Rev. C 4, 934 (1971).

11. E. Cereda et al., Phys. Rev. C 26, 1941 (1982).

12. E. Fretwurst et al., Nucl. Phys. A 468, 247 (1987).

13. P. Lambropoulos et al., Nucl. Phys. A 201, 1 (1973).

14. M. Divadeenam et al., Dissert. Abst. B 28, 3834 (1968).

15. M.V. Pasechnik et al., 5th All Union Conf. on Neutron Physics, Kiev, USSR, 15-19 Sept. 1980 (1980), p. 304.

16. A.B. Smith et al., Nucl. Phys. A 244, 213 (1975).

17. S. Raman et al., At. Data Nucl. Data Tables 78, 1 (2001). 